\title{
Solving path diagrams with EUREKA
}

\author{
JOHN BENJAFIELD \\ Brock University, St. Catharines, Ontario, Canada
}

\begin{abstract}
To solve the path diagrams for a particular causal model, psychologists typically have made use of sophisticated programs such as LISREL. Such programs are difficult for the beginning student to use, and are occasionally awkward or inconvenient even for veteran causal modelers. It would be helpful to such users to have access to a method for conducting causal modeling on a microcomputer without requiring that they have any programming skills. Fortunately, such a procedure is now readily available in the form of EUREKA (1987). This paper describes a way of employing EUREKA so that it is of value not only to naive, but also to sophisticated, users.
\end{abstract}

Causal modeling has become an increasingly important tool in psychology. In 1979, Moreland and Zajonc noted that this form of analysis was less common in psychology than in other social sciences. However, recent developments suggest that psychologists are increasingly interested in this area. Most notably, recent editions of classic statistical texts, such as that by Cohen and Cohen (1983), and new texts, such as that by Loehlin (1987), have begun to stress the importance of causal modeling as a research strategy in psychology. However, with the emergence of causal modeling come a number of problems. One problem is the availability of user-friendly statistical programs, not only for the sophisticated user, but also for the beginning student. This paper describes a program that may be of value to both types of user.

Typically, causal modeling has employed LISREL (Jöreskog \& Sörbom, 1984). Offered as part of SPSS-X, LISREL is more widely used than any other program for causal modeling. However, it is not an easy program to master, and it does not always provide the user with the flexibility he/she needs. As Loehlin $(1987$, p. 72) suggested, there is ample scope for the employment of additional techniques to supplement LISREL. One is Loehlin's (1987, Appendix B) general model fitting program, IPSOL.

Loehlin's (1987, Appendix B) IPSOL program, written in FORTRAN, is accessible to anyone with modest FORTRAN programming skills. However, not all wouldbe causal modelers understand FORTRAN and/or have easy access to a computer that runs FORTRAN. This is especially true of students who are being taught the principles of path analysis and causal modeling. It would be helpful to such users to have access to a method for conducting causal modeling on a microcomputer without requiring that they have any programming skills. Fortunately, such a procedure is now readily available through the use of EUREKA (1987).

Preparation of this paper was facilitated by a grant from the Social Sciences and Humanities Research Council of Canada. The author is grateful to John C. Loehlin for commenting on an earlier draft. Address correspondence to John Benjafield, Department of Psychology, Brock University, St. Catharines, Ontario L2S 3A1, Canada.
EUREKA is an equation-solving program that currently runs on IBM microcomputers and compatibles with at least 384K RAM. The procedures described below were tested on an IBM System/2 Model 50 microcomputer with $640 \mathrm{~K}$ usable RAM, but without a math coprocessor. There is no reason to believe that they will not perform in an equivalent manner on any MS-DOS machine with sufficient RAM.

EUREKA comes with a Wordstar-like editor that is used to prepare an equation file. This file need only contain the equations to be solved. The following is a complete equation file to solve the path diagram given in Figure 1, which has been adapted from Loehlin $(1987$, p. 12):

$$
\begin{aligned}
& c=.50 \\
& a+c * b=.65 \\
& b+c * a=.7
\end{aligned}
$$

Once the equation file has been prepared, the user simply invokes the solution procedure, which requires two keystrokes. EUREKA then provides the solutions for all variables. A report may be saved to disk or routed to a printer.

The equation file in the preceding example was for a just-determined path diagram. It therefore admits of a unique solution, which EUREKA returns in less than a second. Typically, causal modeling is undertaken with

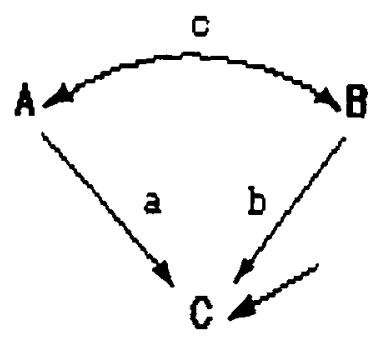

Intercorrelations

Figure 1. Correlation matrix and path diagram to illustrate the solution of path equations in EUREKA. Adapted from Loehlin (1987, p. 12). 


\section{Intercorrelations}

\begin{tabular}{|c|c|c|c|c|c|}
\hline & Ach1 & Ach2 & Amb1 & Amb2 & Amb3 \\
\hline ACn2 & 60 & 1.00 & & & \\
\hline $\mathrm{mb1}$ & .30 & .20 & 1.00 & & \\
\hline $\mathrm{mb} 2$ & .20 & .30 & .70 & 1.00 & \\
\hline $\mathrm{mb3}$ & .20 & .10 & .60 & .50 & 1.00 \\
\hline
\end{tabular}

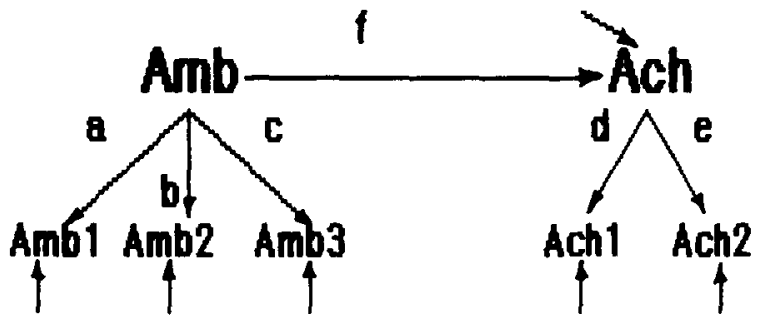

Figure 2. Correlation matrix and path diagrams for Loehlin's $(1987$, p. 245$)$ ambition/achievement model. In the model, there are three measures of ambition and two of achievement.

path diagrams that are overdetermined, such as that given in Figure 2, also adapted from Loehlin (1987, p. 245). These problems do not admit of a unique solution and are usually approached through minimization procedures. Fortunately, such procedures are also available in EUREKA. In fact, it is a simple matter to set up an equation file that allows EUREKA to mimic Loehlin's IPSOL program.

Essentially, IPSOL consists of a set of path equations and a routine that minimizes the differences between the observed correlation coefficients and the correlation coefficients calculated on the basis of the paths. As Loehlin (1987, pp. 54-60) pointed out, there are several criteria that can be adopted for minimizing these differences. One procedure is to attempt to minimize the sum of the squares of the differences between the observed and implied correlations. This is the ordinary least squares criterion, which is also one of the options in LISREL VI (Jöreskog \& Sörbom, 1984).

To obtain the sum of the squares of the differences between the observed and implied correlations, only the fol-

Table 1

Path Coefficients Calculated by EUREKA and LISREL

\begin{tabular}{ccc}
\hline & \multicolumn{2}{c}{ Values } \\
\cline { 2 - 3 } Variables & EUREKA & LISREL \\
\hline $\mathrm{a}$ & .908 & .920 \\
$\mathrm{~b}$ & .785 & .761 \\
$\mathrm{c}$ & .640 & .652 \\
$\mathrm{~d}$ & .834 & .879 \\
$\mathrm{e}$ & .720 & .683 \\
$\mathrm{f}$ & .361 & .356 \\
\hline
\end{tabular}

lowing equation is required (the Cs stand for calculated correlations, and the Os stand for observed correlations):

$$
\mathrm{SUM}=(\mathrm{O} 1-\mathrm{Cl})^{\wedge} 2+\ldots+(\mathrm{On}-\mathrm{Cn})^{\wedge} 2
$$

At this point, all that is required is to minimize SUM. In EUREKA, the minimization procedure is invoked using the reserved word \$min. The following is all that is needed, once SUM has been defined:

\section{$\$ \min ($ SUM)}

Thus, the complete equation file for the model in Figure 2 is

$$
\begin{aligned}
& \mathrm{C} 1=\mathrm{d} * \mathrm{e} \\
& \mathrm{C} 2=\mathrm{a} * \mathrm{f} * \mathrm{~d} \\
& \mathrm{C} 3=\mathrm{b} * \mathrm{f} * \mathrm{~d} \\
& \mathrm{C} 4=\mathrm{c} * \mathrm{f} * \mathrm{~d} \\
& \mathrm{C} 5=\mathrm{a} * \mathrm{f} * \mathrm{e} \\
& \mathrm{C} 6=\mathrm{b} * \mathrm{f} * \mathrm{e} \\
& \mathrm{C} 7=\mathrm{c} * \mathrm{f} * \mathrm{e} \\
& \mathrm{C} 8=\mathrm{a} * \mathrm{~b} \\
& \mathrm{C} 9=\mathrm{a} * \mathrm{c} \\
& \mathrm{C} 10=\mathrm{b} * \mathrm{c} \\
& \mathrm{SUM}=(.6-\mathrm{C} 1)^{\wedge} 2+(.3-\mathrm{C} 2)^{\wedge} 2+(.2-\mathrm{C} 3)^{\wedge} 2 \\
& \quad+(.2-\mathrm{C} 4)^{\wedge} 2+(.2-\mathrm{C} 5)^{\wedge} 2 \\
& \quad+(.3-\mathrm{C} 6)^{\wedge} 2+(.1-\mathrm{C} 7)^{\wedge} 2 \\
& \quad+(.7-\mathrm{C} 8)^{\wedge} 2+(.6-\mathrm{C} 9)^{\wedge} 2 \\
& \quad+(.5-\mathrm{C} 10)^{\wedge} 2
\end{aligned}
$$

\section{$\$ \min ($ SUM)}

A comparison of the path coefficients calculated by EUREKA and those calculated by LISREL is given in Table 1 . These values are clearly quite close to each other. EUREKA is also able to solve most of the problems in Loehlin (1987), as well as the path equations implied by the well-known causal models given by Moreland and Zajonc (1979; Zajonc, 1980). In all these cases, EUREKA yields path coefficients that are excellent approximations of those provided by LISREL and IPSOL.

EUREKA has some very useful options that allow the user to specify the initial values given to variables, and to specify the range of values through which it will search during iteration. Specifying the allowable ranges of parameters is often useful, and this capability is not available in LISREL. The only problems that EUREKA cannot tackle are those containing more than 20 unknowns or more than 20 equations. Although this is a serious restriction, it does not preclude using the program for teaching purposes or for some small problems using real data. 
Obviously, EUREKA is not powerful enough to serve as a competitor for LISREL. Nevertheless, EUREKA does provide a very useful tool for exploratory model fitting, since it is relatively inexpensive (approximately $\$ 100$ U.S.) and very easy to use. The serious investigator can use EUREKA for exploratory work prior to full-fledged model testing with a program such as LISREL. Indeed, for some relatively small models, EUREKA may be all that is needed. Another potential beneficiary of EUREKA is likely to be the graduate student, who can explore a variety of modeling techniques using this program.

Several authors (e.g., Bentler \& Weeks, 1985) have called for statistical software that allows a problem to be represented in a way that the user finds relatively easy and natural. In several ways, EUREKA is representative of an emerging class of microcomputer software that sets a new standard of user-friendliness.

\section{REFERENCES}

Bentler, P. M., \& Weks, D. G. (1985). Some comments on stuctural equation models. British Journal of Mathematical \& Statistical Psychology, 38, 120-121.

COHEN, J., \& COHEN, P. (1983). Applied multiple regression/correlation analysis for the behavioral sciences (2nd ed.). Hillsdale, NJ: Erlbaum. EUREKA: The Solver [Computer program]. (1987). Scott's Valley, CA: Borland International.

JöRESKOG, K. G. , \& SöRBOM, D. (1984). LISREL VI: User's guide (3rd ed.). Mooresville, IN: Scientific Software.

LoEHLIN, J. C. (1987). Latent variable models. Hillsdale, NJ: Erlbaum. Moreland, R. L. \& Zajonc, R. B. (1979). Exposure effects may not depend on stimulus recognition. Joumal of Personality \& Social Psychology, 37, 1085-1089.

ZajonC, R. B. (1980). Feeling and thinking: Preferences need no inferences. American Psychologist, 35, 151-175.

(Manuscript received September 29, 1987: revision accepted for publication February 2, 1988.) 